e-migrinter

e-Migrinter

$14 \mid 2016$

L'ajustement méthodologique comme fabrique critique du savoir dans les études migratoires

\title{
Le contrôle migratoire en Croatie
}

Outils pour approcher les territoires de la frontière extérieure de l'Union européenne

Morgane Dujmovic

\section{OpenEdition}

\section{Journals}

Édition électronique

URL : https://journals.openedition.org/e-migrinter/738

DOI : $10.4000 /$ e-migrinter.738

ISSN : 1961-9685

\section{Éditeur}

UMR 7301 - Migrinter

Référence électronique

Morgane Dujmovic, «Le contrôle migratoire en Croatie », e-Migrinter [En ligne], 14 | 2016, mis en ligne le, consulté le 20 mai 2021. URL : http://journals.openedition.org/e-migrinter/738 ; DOI : https:// doi.org/10.4000/e-migrinter.738

Ce document a été généré automatiquement le 20 mai 2021.

Tous droits réservés 


\section{Le contrôle migratoire en Croatie}

Outils pour approcher les territoires de la frontière extérieure de l'Union européenne

Morgane Dujmovic

\section{NOTE DE L'AUTEUR}

Cette article issu de la présentation d'un poster scientifique. Morgane Dujmovic est doctorante contractuelle au sein de l'UMR 7303 TELEMMe (Temps, Espaces, Langages, Europe Méridionale - Méditerranée) de l'Université d'Aix-Marseille. Elle est invitée scientifique du Département de Géographie à la Faculté des Sciences de l'Université de Zagreb. Ses recherches sont financées par un contrat doctoral de l'École doctorale 355 «Espaces, Cultures, Sociétés ». Ces notes sont issues d'une recherche en thèse de Géographie menée sous la direction de Pierre Sintès (UMR TELEMMe). Les résultats intermédiaires présentés ici n'auraient été atteignables sans le soutien de Laura Šakaja (Département de Géographie de Zagreb), des différents enquêtés, comme du ministère de l'Intérieur croate qui a permis un accès à certains lieux de l'enquête. De chaleureux remerciements vont également à Pierre Sintès, Mike Gadras et Josepha Milazzo pour leurs relectures minutieuses et attentives.

\section{Le poster scientifique : une présentation synthétique et évolutive des enjeux de la thèse}

L'appel à communication de la Journée jeunes chercheurs du laboratoire MIGRINTER de juin 2014 m'a donné l'occasion d'élaborer un poster scientifique afin de présenter une synthèse des résultats préliminaires de ma recherche en thèse, tout en ménageant lisibilité et esthétique qui sont les règles de ce type d'exercice. Il m'a aussi permis de clarifier mon positionnement, tant pour ce qui concerne ma discipline (la géographie sociale et politique) que du point de vue méthodologique et technique : il s'agissait de penser « en grand", pour un format de quatre-vingt centimètres sur cent, tout en 
donnant la priorité à une présentation visuelle des données. Toutefois, la version initiale exposée lors de la journée de lancement du Réseau Migrations comportait encore trop de texte: à l'origine, l'unique illustration était la carte centrale de la version actuelle du poster présentée dans ce numéro spécial. Depuis, le texte a été remodelé en support visuel, alors que le résumé du poster s'est enrichi. L'enjeu du poster est en effet double: une fois sa réalisation menée à terme, il s'agit d'en faire une présentation dynamique qui « capte le regard » et incite celui ou celle qui l'observe à « s'approcher » physiquement afin d'engager la discussion.

2 Je reprendrai ici plusieurs éléments du poster qui m'ont permis de proposer une « boîte à outil analytique » (Darley, 2010, 74) au fondement d'une étude sur la question du contrôle migratoire en Croatie, d'abord dans le contexte particulier de la candidature et de l'adhésion du pays à l'Union européenne (2000-2013) ${ }^{1}$, puis durant la phase d'implantation des dispositifs de l'acquis Schengen, dans la perspective d'intégration dans cet espace réglementaire (2013-2016).

Figure $\mathrm{n}^{\circ} 1$ : Poster scientifique - Le contrôle migratoire en Croatie, construire la frontière extérieure de l'Union Européenne

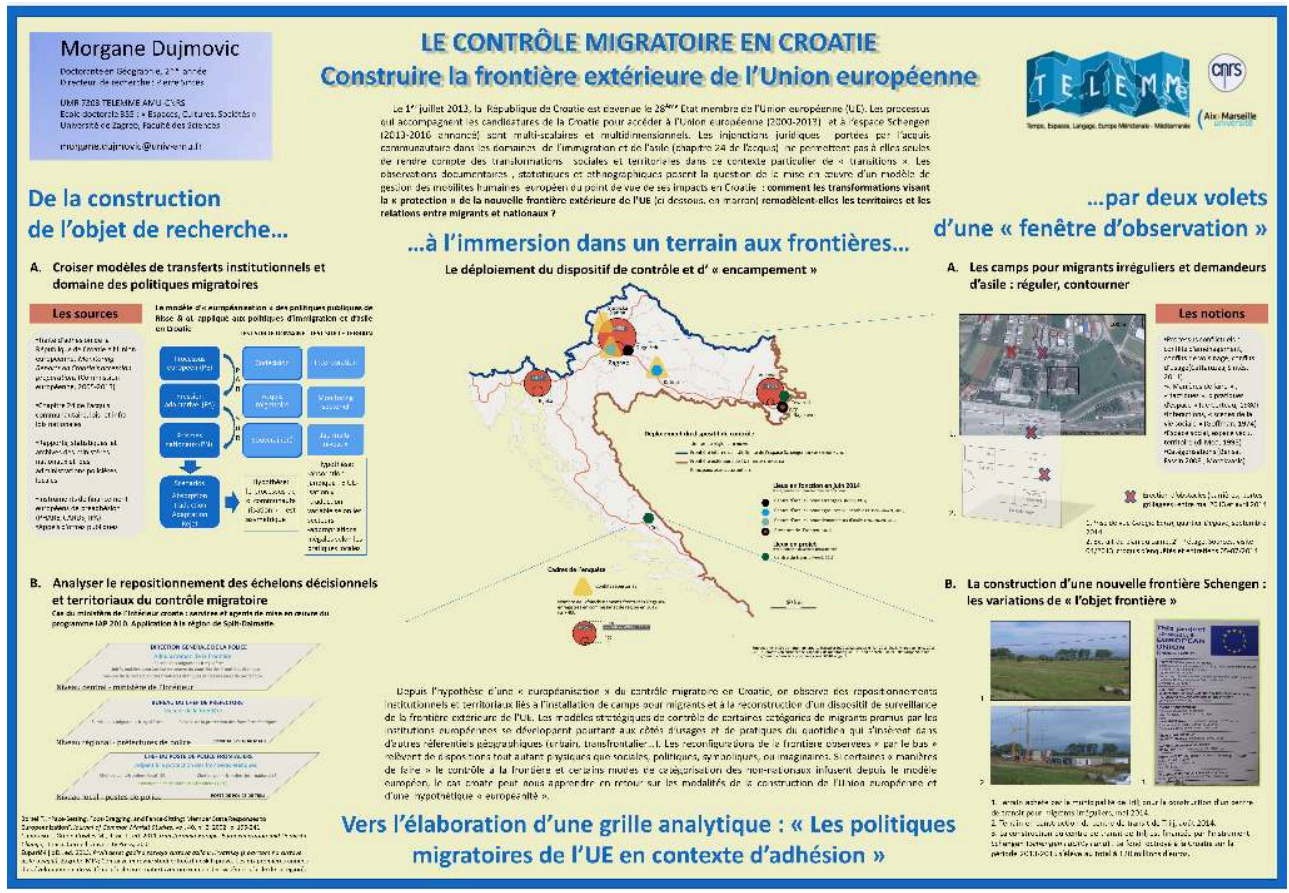

Source : Morgane Dujmovic, 2014.

\section{Une plongée à la frontière : des territoires multidimensionnels et multi-scalaires}

3 Les différents éléments du poster se disposent autour d'une "carte de terrain" centrale (commentée en sous-point 1 ci-après), à partir de laquelle sont projetés les quatre principaux axes de mon travail. Il s'agit de rendre compte des allers-retours progressifs entre trois éléments constitutifs de mes recherches: l'observation documentaire et théorique (partie gauche, en haut du poster ; sous-point 2 ci-après), le travail sur les acteurs et les groupes d'études (partie gauche, en bas du poster; sous- 
point 2 ci-après), et la compilation de données empiriques par le biais d'enquêtes ethnographiques « par le bas » (partie droite du poster ; sous-point 3 ci-après).

\section{La carte de l'objet « encampement », carte de terrain}

4 La carte centrale représente d'une part le déploiement du dispositif de contrôle migratoire. D'autre part, elle rend compte d'un terrain d'enquête construit à partir de résultats empiriques qui témoignent d'un espace d'investigation discontinu et multisitué (Marcus, 1995 ; Cortes, Pesche, 2013)².

5 Les dispositifs de contrôle sont mis en place à la frontière territoriale que l'État croate partage avec ses voisins des Balkans non membres de l'Union européenne (UE) : Serbie, Bosnie-Herzégovine et Monténégro [figuré marron de la carte]. Il s'agit depuis 2013 de la frontière extérieure de l'Union européenne, appelée à devenir, dans un avenir proche, une nouvelle limite de l'espace Schengen (Conseil de l'UE, 2011, art. 31-4, 59)3. Dans un tel contexte, l'étude s'intéresse aux modalités du contrôle de certains groupes de migrants pour lesquels l'accès aux territoires des États membres est restreint voire interdit: les "migrants illégalisés $»^{4}$ et les demandeurs d'asile originaires de pays extérieurs à l'Union européenne, qu'ils soient ou non originaires des Balkans (Morokvasić-Muller, 2001 ; Clochard, 2007).

Du fait d'un dispositif d'accueil très institutionnalisé et centralisé par le ministère de l'Intérieur croate (Ministarstvo Unutarnjih Poslova - MUP), la plupart des migrants sont placés dans des camps gérés par cet acteur étatique. Chacun de ces lieux procède de formes d'assignation résidentielle, que leur fonction soit rétentionnaire ou humanitaire (Bernardot, 2008; Migreurop, 2012). La seconde partie de la légende présente une typologie chronologique et thématique de ce processus "d'encampement »: on y voit apparaître tous les indices de l'installation progressive d'un dispositif de camps qui est, en parallèle, «mis aux normes » de l'UE au cours du processus d'adhésion (Baričević, 2013, 150-161). L'espace discontinu formé par les camps pour migrants apparaît in fine comme une forme particulière de l'objet-frontière, qui est à la fois interne, « pixellisée » et « réticulaire » (Arbaret-Schulz, 2008 ; Bigo, 2011).

7 Ces lieux sont multidimensionnels: d'espace-enjeu dans le cadre des négociations d'adhésion, à espace-instrument du pouvoir permettant la fixation de migrants, les camps sont aussi des espaces-objets ou des espaces-médiateurs (Cattaruzza, Sintès, 2011 ; Di Méo, 1998, 2014) dans les conflits qui éclatent au sujet de leur existence ou de leur perduration [figurés triangulaires jaunes]. Ils se présentent comme les principaux supports de la coprésence des migrants et des nationaux en Croatie en même temps qu'ils sont produits par le jeu des interactions «face à face " entre indigènes et étrangers (Goffman, 1974, 7 ; Bigo, Bocco, Piermay, 2009).

8 Un autre indice de la présence de migrants irréguliers se trouve dans les statistiques diffusées par le MUP, qui font état d'arrestations aux frontières pour motif de "franchissement irrégulier de frontières étatiques" [figurés circulaires rouges]. À chaque étape du dénombrement policier des migrants irréguliers et des demandeurs d'asile correspond donc une procédure spatialisée, ce qu'illustrent les flèches qui jaillissent de la carte vers les autres éléments du poster. 


\section{Univers théoriques et relationnels : le document et les enquêtés}

9 Parmi les nombreuses études qui portent sur les processus «d'européanisation » (Olsen, 2002; Radaelli, 2002), certaines peuvent être testées dansle domaine migratoire. Le modèle théorique développé par Risse \& al. et présenté dans le poster, "permet d'évaluer l'impact du processus européen, médiatisé sous la forme d'une pression adaptative diversement intégrée par les prismes nationaux» (Saurugger, Surel, 2006, 189). Ces processus de transferts de politiques publiques sont visibles aux différents échelons nationaux et locaux du MUP, - ministère qui détient un rôle central dans l'administration des camps, comme de la frontière. La mise en œuvre de dispositifs techniques et juridiques de contrôle de la frontière dans le cadre de l'intégration européenne est illustrée par un diagramme des acteurs qui ont été opérants dans le programme de préadhésion IAP en $2010^{5}$, aux différents niveaux de la Direction générale de la police - depuis l'Administration de la frontière, jusqu'aux agents de la police aux frontières. Le travail de recoupement des données provient de plusieurs niveaux de documentation institutionnelle (communautaire, nationale, locale), du dépouillement d'archives du MUP et d'entretiens réalisés auprès d'individus qui interagissent dans et autour des camps.

\section{Univers empirique : l'« œil et la parole $»^{6}$ autour des camps}

L'un des lieux d'enquête se déploie aux abords et à l'intérieur d'un centre d'accueil pour demandeurs d'asile situé dans la banlieue Sud de Zagreb. Dans la terminologie institutionnelle, la désignation du lieu signifie "refuge» (prihvatilište), alors que les individus qui y sont placés privilégient l'appellation éloquente de "camp ", voire de " prison », comme en témoigne l'extrait d'entretien ci-après :

11 L'hôtel Porin, enfin l'ancien hôtel, c'est pire qu'un camp fermé (...). Au camp tu dors, tu manges, tu dors (...). Ils ont installé des portes en grillage à chaque étage, comme dans une prison (entretien du 10/05/2015, réalisé auprès d'un migrant placé au camp de Zagreb).

En effet, la comparaison des enquêtes menées entre 2013 et 2014 laisse apparaitre des processus de fermeture à l'intérieur même de ce lieu, comme dans son environnement urbain [croix rouges] $]^{7}$. Les différents obstacles à la mobilité qui sont érigés impactent les modalités du contact entre les demandeurs d'asile et les résidents, amenant à des tactiques de contournement renouvelées (De Certeau, 1980).

Le second cas d'étude présenté sur le poster correspond à la municipalité de Trilj, en Dalmatie, qui jouxte la région d'Herzégovine en Bosnie. Deux clichés retracent l'évolution, entre mai et août 2014, du chantier d'un centre de transit pour migrants irréguliers, dont la construction est financée en majeure partie par «l'instrument Schengen temporaire» (Schengen Facility Fund). La présence de ce camp réactive l'histoire migratoire de la ville qui, depuis l'époque de l'Empire ottoman, est perçue comme un espace de transit - selon l'image du « pont » qui enjambe la rivière Cetina, vers ou depuis l'Orient (Cvijić, 1918; Todorova, 2011, 37). L'existence d'un «espace vécu (Frémont, 1976), - comme lieu traditionnellement «de passage» ou «de transit » -, s'incarne chez les habitants de la localité comme chez les décideurs locaux. Pour préparer l'installation du camp, les autorités municipales ont d'ailleurs argué de cette fonction de "transit», pour signifier que la présence de migrants serait « uniquement de courte durée $»^{8}$. Dans ce cas, nous n'avons pas observé de réactions 
propres au NIMBY (Not in My Backyard) ${ }^{9}$, contrairement à ce qui a pu être constaté dans d'autres municipalités croates, où des projets de camps ont été abandonnés du fait d'importantes oppositions locales (Petrović, 2013). À l'échelle locale dans le cas de Trilj, la construction du camp est avant tout perçue comme un débouché économique potentiel, en contexte de chômage élevé (DZS, 2013, 157).

\section{Des études de cas à la grille d'analyse : le va-et-vient empirie-théorie}

14 Avec cette affiche, j'ai cherché à représenter différentes dimensions de la frontière et diverses échelles du contrôle à trois étapes-clés du protocole de recherche [en bleu]: depuis la construction de l'objet de recherche, jusqu'à la construction d'un terrain. Ce dernier m'a permis d'analyser, à partir du contrôle migratoire, les évolutions des territoires de la frontière, ce par des phases d'immersion plaçant la focale sur deux groupes d'études de cas, comme autant de volets d'une même "fenêtre d'observation ». Cette démarche vise l'élaboration d'une grille d'analyse des modalités du contrôle migratoire en contexte d'adhésions politiques multiples.

Pour chacun de ces cas d'étude, - aux côtés d'un modèle stratégique du contrôle migratoire qui correspond à des engagements de l'État croate vis-à-vis de l'Union européenne -, des catégories, des référentiels, des manières de faire, et des pratiques de la frontière ou du territoire se rapportent à d'autres niveaux et dimensions (l'urbain, cas du camp de Zagreb ; le transfrontalier, cas du camp de Trilj). L'exemple croate peut donc nous instruire sur les modalités de la construction d'un espace de «librecirculation » européen.

\section{BIBLIOGRAPHIE}

Arbaret-Schulz, Christiane (2008) La question du continu et du discontinu à l'épreuve de la dimension technique des sociétés, in Fréderic, A. ; Génin, A. (Dir.) Continu et discontinu dans l'espace géographique, Tours, Presses universitaires François-Rabelais, pp. 409-416 (Perspectives, Villes et Territoires) [réf. du 27 février 2015] [Disponible sur Internet] .

Baričević, Vedrana (2013) Europeanization of Asylum System and Refugee Protection: Croatian Asylum and Migration Policies, Ljubljana, Université de Ljubljana ; Faculté des sciences sociales, 341 p. Th. Doct. : Géogr. : Université de Ljubljana : 2013.

Bauder, Harald (2013) Why We Should Use the Term Illegalized Immigrant, RCIS Research Brief, $\mathrm{n}^{\circ} 1,7 \mathrm{p}$.

Bernardot, Marc (2008) Camps d'étrangers, Vulaines-sur-Seine, Éditions du croquant, 223 p. (TERRA).

Bigo, Didier (2011) Frontières, territoire, sécurité, souveraineté, CERISCOPE dossier Frontières, [réf. du 06 mars 2015] [Disponible sur Internet]. 
Bigo, Didier ; Bocco, Riccardo ; Piermay, Jean-Luc (2009) Logiques de marquage : murs et disputes frontalières, Cultures \& Conflits, $n^{\circ}$ 73, pp. 7-13 [réf. du 01 janvier 2013] [Disponible sur Internet].

Cattaruzza, Amaël ; Sintès, Pierre (2011) Géographie des conflits, Paris, Bréal, 221 p. (Amphi Géographie Capes Agrégation).

Clochard, Olivier (2007) Le jeu des frontières dans l'accès au statut de réfugié : une géographie des politiques européennes d'asile et d'immigration, Poitiers, Université de Poitiers, $471 \mathrm{p}$.

Th. Doct : Géogr. : Poitiers : 2007.

Conseil européen de Santa Maria da Feira (2000) Conclusions de la Présidence, [réf du 02 avril 2015] [Disponible sur Internet]..

Cortes, Geneviève ; Pesche, Denis (2013) Territoire multisitué, L'Espace géographique, vol. 42, $\mathrm{n}^{\circ} 4$, pp. 289-292, [réf. du 05 mars 2015] [Disponible sur Internet].

Council of the European Union (2011) Accession Treaty: Treaty concerning the accession of the Republic of Croatia, Bruxelles, 272 p. [réf. du 12 octobre 2014] [Disponible sur Internet].

Cvijić, Jovan (1918) La péninsule balkanique : géographie humaine, Paris, Armand Colin, 528 p.

Darley, Mathilde (2010) La construction du problème de l'immigration en République Tchèque : l'action publique sous influence européenne ?, Politique européenne, $\mathrm{n}^{\circ}$ 31, pp. 65-90.

De Certeau, Michel (1990 ) L'invention du quotidien. 1. Arts de faire, Paris, Gallimard, 350 p. (Folio Essais).

Di Méo, Guy (1998) Géographie sociale et territoires, Paris, Éditions Nathan, 320 p.

Di Méo, Guy (2014) Introduction à la géographie sociale, Paris, Armand Colin, 189 p. (Cursus).

Državni Zavod za Statistiku (2013) Statistical Yearbook of the Republic of Croatia 2013, Zagreb, DZS, $584 \mathrm{p}$.

Frémont, Armand (1976) La région, espace vécu, Paris, P.U.F., 223 p. (SUP).

Goffman, Erving (1974) Les rites d'interaction, Paris, Les éditions de minuit, 225 p.

Marcus, George (1995) Ethnography in/of the World System : The Emergence of Multi-Sited Ethnography, Annual Review of Anthropology, vol. 24, n 1, pp. 95-117.

Migreurop ; Clochard, Olivier (Dir.) (2012) Atlas des migrants en Europe. Géographie critique des politiques migratoires, Paris, Armand Colin, $143 \mathrm{p}$.

Ministarstvo Unutarnjih Poslova (2013) Statistički pregled. Temeljnih sigurnosnih pokazatelja $i$ rezultata rada u 2012 Godini, [Examen statistique. Indicateurs de sécurité fondamentaux et résultats d'activités en 2012] Zagreb, 147 p., [réf. du 03 avril 2015] [Disponible sur Internet].

Morokvasić-Muller, Mirjana (2001) Balkans : les exclus de l'élargissement, Hommes et migrations, $\mathrm{n}^{\circ} 1230$, pp. 81-93.

Olsen, Johan (2002) The Many Faces of Europeanization, JCMS : Journal of Common Market Studies, vol. 40, n 5, pp. 921-952.

Petrović, Nikola (2013) Otpori izgradnji prihvatilišta za tražitelje azila : slučaj Stubičke Slatine in Župarić-Iljić, D.(Dir.) Prvih deset godina razvoja sustava azila u Hrvatskoj (s osvrtom na sustave azila u regiji), [Les dix premières années du développement du système d'asile en Croatie (avec un examen des systèmes d'asile de la région)], Zagreb, IMIN ; Centar za mirovne studije ; Kuća ljudskih prava, $280 \mathrm{p}$. 
Radaelli, Claudio (2002) The Domestic Impact of European Union Public Policy : Notes on Concepts, Methods, And the Challenge of Empirical Research, Politique européenne, $\mathrm{n}^{\circ} 5$, pp. 105-136.

Saurugger, Sabine ; Surel, Yves (2006) L'européanisation comme processus de transfert de politique publique, Revue internationale de politique comparée, vol. 13, n 2, pp. 179-211.

Todorova, Maria (2011) Imaginaire des Balkans, Paris, Éd. EHESS, 352 p. (En temps \& lieux).

\section{NOTES}

1. Le $1^{\text {er }}$ juin 2000 le Conseil européen de Feira marque une avancée dans le processus de candidature de la Croatie qui obtient à cette date le statut de «candidat potentiel » comme les autres États des «Balkans occidentaux » inclus dans le Processus de Stabilisation et d'Association. 2. Cortes et Pesche définissent le concept de "territoire multisitué » comme "un ensemble de lieux discontinus constitutif d'un espace fonctionnel et vécu (pratiques, activités, représentations), voire organisationnel, dès lors que sont en jeu des processus d'action, de gestion et de coopération ». Cet ensemble "se construit, se lit, se décrypte à la façon d'un kaléidoscope, c'est à dire dans la pluralité et la variation de la situation relative et relationnelle de chacun des lieux et des réseaux qui le constituent » (Cortes, Pesche, 2013, 290).

3. Aucune date de candidature à l'entrée dans l'espace Schengen n'est arrêtée officiellement et les prévisions en la matière varient en fonction des différents interlocuteurs. Néanmoins, le traité d'adhésion de la Croatie, entré en vigueur le $1^{\mathrm{er}}$ juillet 2013, prévoit une période d'utilisation des aides financières transitoires de trois ans, suivie d'une période de six mois pour fournir un rapport de progrès, ce qui ramène la candidature potentielle de la Croatie à l'espace Schengen à la fin de 2016.

4. L'expression « migrant illégalisé » se démarque de celle du " migrant illégal », car elle invite à déchiffrer les processus par lesquels est forgée l'illégalité de certains migrants par la mise en œuvre de politiques migratoires restrictives (Bauder, 2013).

5. L'Instrument d'Aide de Préadhésion (IAP) est l'instrument financier fourni aux États candidats potentiels et candidats dans le cadre de leur adhésion à l'UE, à partir de 2007.

6. Les moyens d'investigation cités (le document, l'œil et la parole) sont empruntés à Armand Frémont (1976).

7. Les terrains d'enquête menés à $n$ et à $n+1$ avaient pour objectif d'étudier l'ensemble des processus de territorialisation se ramenant aux camps pour migrants, y compris ceux de type "semi-ouverts" qui sont destinés aux demandeurs d'asile. Ces terrains ont consisté en des observations directes in situ, des entretiens, des focus groups, des observations des « espaces de vie » avec les différents employés du camp, avec les individus placés en camp et avec les résidents des localités environnantes.

8. D'après un entretien mené avec l'adjoint au maire de Trilj, le 07/05/2014.

9. L'expression NIMBY signifie "Not In My Backyard». Elle est initialement utilisée par les aménageurs américains pour dévaloriser les oppositions locales aux projets d'urbanisme. Pour d'autres observateurs, le NIMBY serait toutefois « le signe d'un besoin inassouvi de consultation, d'information, d'implication dans la phase préparatoire de la prise de décision », et pourrait être à l'origine de dynamiques sociales positives (Cattaruzza, Sintès, 2011). 
INDEX

Mots-clés : frontière, contrôle des frontières, étude de cas

Index géographique : Croatie

\section{AUTEUR}

MORGANE DUJMOVIC

Doctorante en Géographie, Université d'Aix-Marseille (TELEMMe)

morgane.dujmovic@univ-amu.fr 